\title{
Reconsidering Guidelines on the Use of Pneumococcal Vaccines in Adults 65 Years or Older
}

\section{Citation}

Hochman, Michael, and Pieter A. Cohen. 2015. "Reconsidering Guidelines on the Use of Pneumococcal Vaccines in Adults 65 Years or Older." JAMA Internal Medicine 175 (12)

(December 1): 1895. doi:10.1001/jamainternmed.2015.5689.

\section{Published Version}

10.1001/jamainternmed.2015.5689

\section{Permanent link}

http://nrs.harvard.edu/urn-3:HUL.InstRepos:34902782

\section{Terms of Use}

This article was downloaded from Harvard University's DASH repository, and is made available under the terms and conditions applicable to Other Posted Material, as set forth at http:// nrs.harvard.edu/urn-3:HUL.InstRepos:dash.current.terms-of-use\#LAA

\section{Share Your Story}

The Harvard community has made this article openly available.

Please share how this access benefits you. Submit a story.

Accessibility 


\section{Michael Hochman, MD, MPH \\ Department of Medicine, David Geffen School of Medicine, UCLA (University of California, Los Angeles).}

Pieter A. Cohen, MD Department of Medicine, Harvard Medical School, Boston, Massachusetts.

Viewpoint

\author{
Corresponding \\ Author: Michael \\ Hochman, MD, MPH, \\ Department of \\ Medicine, David Geffen \\ School of Medicine, \\ UCLA, 2040 Camfield \\ Ave, Los Angeles, CA, \\ 90405 (meh1979 \\ @gmail.com).
}

The recent adult pneumococcal vaccine recommendations issued by the Advisory Committee on Immunization Practices (ACIP) and endorsed by the Centers for Disease Control and Prevention are well intentioned but misguided, complex, and not ready for widespread adoption. ${ }^{1}$

In August 2014, the ACIP ${ }^{1}$ recommended the newer pneumococcal conjugate vaccine 13 (PCV13) (\$152 per dose $^{2}$ ) for adults 65 years or older followed 6 to 12 months later by the older pneumococcal polysaccharide vaccine 23 (PPSV23) ( $\$ 72$ per dose ${ }^{2}$ ) (Box). The PCV13 covers 12 of 23 pneumococcal serotypes in PPSV23 plus an additional serotype, and contains conjugate protein, which renders a more robust immune response in individuals with immature or impaired immune systems. In the United States, the conjugate vaccine has been recommended for several years for young children and for patients who are immunocompromised.

In its 2014 report describing the new guidelines, the ACIP ${ }^{1}$ prominently cited the Community-Acquired Pneumonia Immunization Trial in Adults (CAPiTA), a randomized trial of PCV13 among 85000 adults 65 years or older, but it was not until March 2015 that the results of CAPiTA were published. ${ }^{4}$

To place the CAPiTA trial in context, it is important to recognize that the older PPSV23 vaccine has established efficacy against pneumococcal disease. A recent Cochrane Collaboration analysis ${ }^{5}$ of 11 randomized trials involving 36489 participants 16 years or older concluded that PPSV23 was $74 \%$ ( $95 \% \mathrm{Cl}, 55 \%-86 \%$ ) effective against invasive pneumococcal disease and $74 \%$ ( $95 \% \mathrm{Cl}, 54 \%-85 \%)$ effective against confirmed pneumococcal pneumonia.

However, PPSV23 is far from perfect. There have yet to be any high-quality randomized trials focusing on PPSV23 in elderly individuals, and the available data on the elderly population are at times conflicting (although overall, lower-quality observational data ${ }^{5}$ suggest $68 \%$ [95\% Cl, 53\%-78\%] effectiveness against invasive pneumococcal disease in elderly individuals). Studies ${ }^{1}$ have also raised questions about the durability of the immune response to PPSV23. In addition, since Pneumococcus is responsible for only a fraction of the cases of pneumonia, its effect on overall pneumonia rates, including nonpneumococcal pneumonia, is modest (estimated efficacy, 28\%; 95\% Cl, 7\%-44\%). ${ }^{5}$

Despite these limitations, vaccination with PPSV23 is the standard of care for individuals 65 years or older in many countries, including the United States. Thus, the key issue is whether PCV13 adds value when given instead of, or in addition to, PPSV23.

Unfortunately, CAPiTA did not answer the key question. Pfizer, the manufacturer of PCV13, conducted the study in the Netherlands, ${ }^{4}$ where adults do not routinely receive PPSV23, and compared PCV13 with placebo. The PCV13 vaccine performed well relative to placebo, although not overwhelmingly so. During the 4-year study, the efficacy of PCV13 against invasive pneumococcal disease was a modest $52 \%(95 \% \mathrm{Cl}, 22 \%-71 \%)$, and against confirmed pneumococcal pneumonia, its efficacy was just $31 \%$ (95\% Cl, 10\%-47\%). Moreover, vaccination with PCV13 did not significantly reduce the overall rate of community-acquired pneumonia, nor did it reduce mortality due to pneumococcal disease. Because CAPiTA included only adults 65 years and older and the Cochrane Review ${ }^{5}$ included individuals as young as 16 years, it would be misleading to directly compare efficacy rates for PCV13 in CAPiTA with the efficacy rates for PPSV23.

Another notable limitation of CAPiTA is that it took place in the Netherlands before childhood pneumococcal vaccination was well established. Because children are important reservoirs for Pneumococcus, childhood vaccination reduces pneumococcal disease in all age groups (ie, herd immunity). Since routine childhood pneumococcal vaccination began in the United States in 2000, there has been a $90 \%$ reduction in vaccine-type invasive pneumococcal disease and a $50 \%$ reduction in overall disease among all age groups. ${ }^{6}$ Childhood vaccination in the Netherlands did not begin until 2006, which was 2 years before CAPITA started and not in sufficient time for maximal herd protection. Thus, the results likely overstate the benefits of PCV13 relative to settings with longstanding childhood PCV13 vaccination.

Still, the ACIP moved forward with its recommendation to include both PCV13 and PPSV23 in the vaccination routine, promising to reevaluate this recommendation in 2018. The committee chose this reevaluation date because, although US children began receiving a pneumococcal conjugate vaccine covering 7 serotypes (PCV7) in 2000, the newer PCV13, which covers 6 additional strains, was not routinely used until 2010. By 2018 , herd immunity for these additional 6 strains should be well established.

In the meantime, we are concerned that the guidelines from the ACIP and endorsed by the CDC will be com- 
Box. The ACIP/CDC Adult Pneumococcal Vaccine Recommendations $^{\mathrm{a}}$

Pneumococcal vaccine-naive adults 65 years or older should receive a dose of PCV13 first, followed by a dose of PPSV23 12 or more months afterward. ${ }^{\mathrm{b}}$

Adults 65 years or older who have previously received 1 or more doses of PPSV23 also should receive PCV13 if they have not yet received it. A dose of $\mathrm{PCV} 13$ should be given 1 or more years after receipt of the most recent PPSV23 dose, and a subsequent PPSV23 dose should be given 12 or more months after PCV13 and 5 or more years after the most recent dose of PPSV23.

Abbreviations: ACIP, Advisory Committee on Immunization Practices; CDC Centers for Disease Control and Prevention; PCV13, pneumococcal conjugate vaccine 13; PPSV23, pneumococcal polysaccharide vaccine 23

${ }^{\text {a }}$ Adapted from Tomczyk et al. ${ }^{1}$

${ }^{\mathrm{b}}$ In June $2015,{ }^{3}$ the ACIP changed the interval of the subsequent PPSV23 dose to 12 months rather than 6 to 12 months, as originally recommended.

plex and costly to implement. Because repeated pneumococcal vaccination at intervals of less than 12 months may be detrimental to the immune response, ${ }^{7}$ the ACIP recommends specific dosing intervals (Box), requiring complex workflow changes at medical offices where the vaccine is administered. These dosing intervals have already required modification by the ACIP due to their complexity. ${ }^{3}$ This creates challenges for primary care clinics that are already strained by other quality and regulatory requirements, particularly since the new vaccine workflows may be adjusted again in a few years. The new vaccine schedule changes may also adversely affect adherence to vaccination. In addition, at $\$ 152$ per dose, adding PCV13 to the existing vaccination schedule substantially increases overall vaccination costs (although this cost will be covered by insurers in most cases).

Others share our concerns. A recent clinical guidelines synopsis about the 2014 ACIP recommendations ranked them as "poor/ fair" with respect to "establishing evidence foundations and rating strength for each of the guideline recommendations." ${ }^{8(p 719)}$ In addition, the liaison to the ACIP for the American Academy of Family Physicians explained in a statement, "there is no evidence, explanation, or understanding of what happens to people who have had PPSV23 [regarding] how much PCV13 helps in addition." 9

Why did the ACIP make these recommendations? The ACIP correctly points out that PCV13 induces higher antibody levels than PPSV23 in people with weakened immunity, such as individuals 65 years or older. In addition, the clinical efficacy of PPSV23 in that age group is not well established. Finally, there is evidence that priming with PCV13 followed by a PPSV23 booster dose induces higher antibody levels compared with a single dose of either vaccine, although the clinical relevance of this finding is uncertain. ${ }^{7}$

The rationales for the ACIP recommendations are important factors to consider. Nonetheless, this reasoning does not obviate the need for compelling clinical data demonstrating the value of giving PCV13 in addition to PPSV23, particularly in the United States, where herd immunity has substantially reduced the incidence of pneumococcal disease and the added effect of PCV13 is likely to be small.

In our view, a simpler and less costly way to reduce pneumococcal disease would be to improve systems to increase pneumococcal vaccination among children and adults (more than one-third of US adults $\geq 65$ years have not received PPSV23). ${ }^{10}$ Pneumococcal diseases remain an important health challenge. Health care professionals and public health officials should not lose sight of the most straightforward way to address this challenge.
ARTICLE INFORMATION

Published Online: October 26, 2015. doi:10.1001/jamainternmed.2015.5689.

Conflict of Interest Disclosures: None reported.

\section{REFERENCES}

1. Tomczyk S, Bennett NM, Stoecker C, et al; Centers for Disease Control and Prevention (CDC). Use of 13-valent pneumococcal conjugate vaccine and 23-valent pneumococcal polysaccharide vaccine among adults aged $\geq 65$ years: recommendations of the Advisory Committee on Immunization Practices (ACIP). MMWR Morb Mortal Wkly Rep. 2014;63(37):822-825.

2. Centers for Disease Control and Prevention. Vaccines for Children Program (VFC): CDC vaccine price list. http://www.cdc.gov/vaccines/programs /vfc/awardees/vaccine-management/price-list/. Accessed September 8, 2015.
3. Kobayashi M, Bennett NM, Gierke R, et al. Intervals between PCV13 and PPSV23 vaccines: recommendations of the Advisory Committee on Immunization Practices (ACIP). MMWR Morb Mortal Wkly Rep. 2015;64(34):944-947.

4. Bonten MJ, Huijts SM, Bolkenbaas M, et al. Polysaccharide conjugate vaccine against pneumococcal pneumonia in adults. $N$ Engl $J$ Med 2015;372(12):1114-1125.

5. Moberley S, Holden J, Tatham DP, Andrews RM Vaccines for preventing pneumococcal infection in adults. Cochrane Database Syst Rev. 2013;1: CD000422. doi:10.1002/14651858.CD000422.pub3.

6. Pilishvili T, Lexau C, Farley MM, et al; Active Bacterial Core Surveillance/Emerging Infections Program Network. Sustained reductions in invasive pneumococcal disease in the era of conjugate vaccine. J Infect Dis. 2010;201(1):32-41.

7. O'Brien KL, Hochman M, Goldblatt D. Combined schedules of pneumococcal conjugate and polysaccharide vaccines: is hyporesponsiveness an issue? Lancet Infect Dis. 2007;7(9):597-606.

8. Pisano J, Cifu AS. Use of pneumococcal vaccine in adults. JAMA. 2015;313(7):719-720.

9. American Academy of Family Physicians. ACIP recommends routine PCV13 immunization for adults 65 and older. http://www.aafp.org/news /health-of-the-public/20140827pcv13vote.html. Published August 27, 2014. Accessed September 8, 2015.

10. National Foundation for Infectious Diseases. Pneumococcal disease call to action: protecting older Americans from serious pneumococcal disease: a task force report. April 2012. http://www .adultvaccination.org/professional-resources /pneumococcal-cta/older.pdf. Published April 2012. Accessed September 8, 2015. 